\title{
Left Ventricular Outflow Tract Area After Percutaneous Transseptal Transcatheter Mitral Valve Implantation; A Three-dimensional Transesophageal Echocardiography Study
}

atsushi hayashi ${ }^{1}$, hiroki ikenaga ${ }^{1}$, takafumi nagaura ${ }^{1}$, Jun Yoshida ${ }^{2}$, Florian Rader ${ }^{2}$, moody makar $^{2}$, Tarun Chakravarty ${ }^{3}$, Robert J. Siegel ${ }^{4}$, saibal kar ${ }^{1}$, Raj Makkar ${ }^{5}$, and Takahiro Shiota $^{3}$

${ }^{1}$ Affiliation not available

${ }^{2}$ Cedars-Sinai Medical Center

${ }^{3}$ Cedars Sinai Medical Center

${ }^{4}$ Cedars Sinai Med Ctr

${ }^{5}$ Cedar Sinai Medical Center

January 18, 2021

\begin{abstract}
Background: Left ventricular (LV) outflow tract (LVOT) obstruction increases mortality in patients undergoing transcatheter mitral valve implantation (TMVI) in degenerated bioprostheses, annuloplasty rings, and native mitral valves. We aimed to evaluate the left ventricular outflow tract area after TMVI using 3-diensional (3D) transesophageal echocardiography (TEE) and to investigate the pre-procedural cardiac geometry affects the LVOT area after TMVI. Methods: We retrospectively reviewed echocardiography data in 43 patients who had TMVI. A change in pressure gradient across LVOT from before to after TMVI ([?]PG) and post-procedure 3D cross sectional area (CSA) at the level of the most distal portion of the mitral valve stent that was closest to the LV apex were assessed as evidence of LVOT narrowing. Results: TMVI with the use of balloon-expandable valve system was performed for 24 bioprostheses, 7 annuloplasty rings, and 12 native valves. Compared to patients without increase in LVOT gradient ([?]PG <10 mmHg; n=33), patients with increase in LVOT gradient ([?]PG [?]10 mmHg; n=10) had smaller LV end-systolic volume (LVESV), greater LV ejection fraction (LVEF) and smaller aorto-mitral (AM) angle. CSA at the valve stent distal edge showed strong association with [?]PG $(\mathrm{r}=-0.68, \mathrm{P}<0.0001)$. Only small AM angle was associated with small CSA at the valve stent ventricular edge on multivariable analysis, independent of LVESV and LVEF. Conclusion: Pre-procedural AM angle as well as LVESV and LVEF were associated with LVOT narrowing in patients undergoing transcatheter mitral valve-in-valve, valve-in-ring, and valve-in-native valve implantation. These data may be useful for preprocedural planning.
\end{abstract}

Left Ventricular Outflow Tract Area After Percutaneous Transseptal Transcatheter Mitral Valve Implantation; A Three-dimensional Transesophageal Echocardiography Study

Atsushi Hayashi $\mathrm{MD}^{1}$, Hiroki Ikenaga $\mathrm{MD}^{1}$, Takafumi Nagaura MD ${ }^{1}$, Jun Yoshida MD ${ }^{1}$, Florian Rader $\mathrm{MD}^{1}$, Moody Makar MD2, Tarun Chakravarty $\mathrm{MD}^{3}$, Robert J. Siegel MD${ }^{1}$, Saibal Kar MD³, Raj R. Makkar MD Takahiro Shiota $\mathrm{MD}^{1}$.

${ }^{1}$ Department of Noninvasive Cardiac Laboratory, Cedars-Sinai Smidt Heart Institute, Los Angeles, California.

${ }^{2}$ Department of Anesthesiology, Cedars-Sinai Medical Center, Los Angeles, California.

${ }^{3}$ Department of Interventional Cardiology, Cedars-Sinai Smidt Heart Institute, Los Angeles, California. 


\section{Running head: Narrowed LVOT area after TMVI}

Address for correspondence:

Takahiro Shiota, MD.

Department of Noninvasive Cardiac Laboratory, Cedars-Sinai Smidt Heart Institute, 127 South San Vincente Blvd, A3411, Los Angeles, CA 90048, U.S.A.

Tel: +1-(310)-423-6889, FAX: +1-(310)-423-8571, E-mail: Takahiro.Shiota@cshs.org

\section{Abstract}

\section{Background:}

Left ventricular (LV) outflow tract (LVOT) obstruction increases mortality in patients undergoing transcatheter mitral valve implantation (TMVI) in degenerated bioprostheses, annuloplasty rings, and native mitral valves. We aimed to evaluate the LVOT area after TMVI using 3-diensional (3D) transesophageal echocardiography (TEE) and to investigate the pre-procedural cardiac geometry affects the LVOT area after TMVI.

\section{Methods:}

We retrospectively reviewed echocardiography data in 43 patients who had TMVI. A change in pressure gradient across LVOT from before to after TMVI ([?]PG) and post-procedure 3D LVOT cross sectional area at the level of the most distal portion of the mitral valve stent that was closest to the LV apex were assessed as evidence of LVOT narrowing.

\section{Results:}

TMVI with the use of balloon-expandable valve system was performed for 24 bioprostheses, 7 annuloplasty rings, and 12 native valves. Compared to patients without increase in LVOT gradient ([?]PG $<10 \mathrm{mmHg}$; $\mathrm{n}=33$ ), patients with increase in LVOT gradient ([?]PG [?]10 $\mathrm{mmHg} ; \mathrm{n}=10$ ) had smaller LV end-systolic volume (LVESV), greater LV ejection fraction (LVEF) and smaller aorto-mitral (AM) angle. LVOT area at the valve stent distal edge showed strong association with [?]PG $(r=-0.68, \mathrm{P}<0.0001)$. Only small AM angle was associated with small LVOT area at the valve stent distal edge on multivariable analysis, independent of LVESV and LVEF.

\section{Conclusion:}

Pre-procedural AM angle as well as LVESV and LVEF were associated with LVOT narrowing in patients undergoing transcatheter mitral valve-in-valve, valve-in-ring, and valve-in-native valve implantation. These data may be useful for preprocedural planning.

Keywords: 3D, echocardiography, transcatheter, mitral valve, left ventricular outflow tract

\section{INTRODUCTION}

Percutaneous transseptal transcatheter mitral valve implantation (TMVI) using balloon-expandable valve systems is developing as an alternative to open heart mitral valve (MV) surgery for high-risk surgical patients with degenerated bioprosthetic valves, failed repairs with annuloplasty ring, and native MV insufficiency or stenosis. ${ }^{1-3}$ Although safety and feasibility of this new approach and acceptable one-year outcome after procedure have been reported, ${ }^{4-6}$ there are still several challenges to overcome: stent malposition, embolization, and left ventricular outflow tract (LVOT) obstruction. Therefore, a comprehensive approach or planning to prevent these complications is required. ${ }^{7,8}$

LVOT obstruction following transseptal TMVI in bioprosthetic valves [valve-in-valve (ViV)], surgical MV annuloplasty rings [valve-in-ring (ViR)], and native MV [valve-in-native valve (ViN)] has been recognized early in the development of this technique and increases mortality in these patients. ${ }^{3,9}$ The TMVI device elongates the pre-existing native outflow tract toward the LV apex and creates a new LVOT (neo-LVOT) 
surrounded by surgical valve leaflet or native mitral anterior leaflet and interventricular septum. Accordingly, the geometry and function of the left ventricle, aortic valve, and mitral valve may lead to narrow LVOT area after TMVI. Yoon et al. reported that estimated neo-LVOT area from pre-procedural multidetector low computed tomography (MDCT) could help identify the patients at high risk for LVOT obstruction assessed by peak LVOT gradient. ${ }^{10}$ However, there were limited studies directly assessing the LVOT cross sectional area after TMVI and identifying its association with anatomical/functional factors such as septal hypertrophy, left ventricular (LV) cavity size, LV wall motion, and angulation between the aortic annulus and the mitral annulus (aorto-mitral angle). Notably, the degree of aorto-mitral (AM) angle as an obtuse angle may be closely related to the LVOT narrowing. If the angle would be small, valve stent implanted at the mitral position would project into the LVOT, resulting in the narrowing of the LVOT space. While, if the angle is enough large, valve stent would project to the LV cavity and thus did not alter the LVOT geometry. Three-dimensional transesophageal echocardiography (3D TEE) can contribute to our understanding of the relationships between MV and basal LV wall with the unique morphology of the neo-LVOT space. ${ }^{11}$ Furthermore, this technology is well established in the assessment of dynamic LVOT size in hypertrophic obstructive cardiomyopathy. ${ }^{12}$ Accordingly, we tested the hypothesize that intraprocedural real-time 3D TEE can quantify the LVOT area and that pre-procedural AM angle can be associated with LVOT narrowing in patients undergoing transcatheter mitral ViV, ViR, and ViN implantation.

\section{MATERIALS AND METHODS}

\subsection{Study population}

We reviewed 48 patients who had TMVI with balloon-expandable transcatheter valve systems using Sapien family valves (Sapien XT or Sapien 3; Edwards Lifesciences, Irvine, California) under real-time 3D TEE guidance between March 2014 and October 2018 at Cedars-Sinai Smidt Heart Institute (Los Angeles, California, U.S.A.). Among them, 4 patients with concomitant transcatheter aortic valve replacement (3 for aortic stenosis and 1 for aortic regurgitation) were excluded because it was difficult to assess the change in LVOT gradient from before to after the TMVI procedure using continuous wave Doppler, and 1 with suboptimal image quality were also excluded. A total of 43 patients were included in this study. This study was approved by the Cedars-Sinai institutional review board.

\subsection{Two-Dimensional transthoracic echocardiography}

Standard 2-dimensional (2D) and Doppler transthoracic echocardiography was performed at baseline and after TMVI [30 (9-35) days] using an iE33 ultrasound system (Philips Healthcare, Andover, MA, USA) equipped with a $2.5-\mathrm{MHz}$ transducer. All measurements were made according to the current guideline. ${ }^{13}$ The peak LVOT velocity was measured with continuous Doppler echocardiography in apical LV long-axis view, aligning the continuous Doppler beam as parallel as possible to the LVOT jet. The pressure gradient (PG) across LVOT was calculated using simplified Bernoulli equation; $P G=4 \times$ (peak velocity) ${ }^{2}$. The change in $\mathrm{PG}$ across LVOT $(\triangle \mathrm{PG})$ was defined as follows; $\triangle \mathrm{PG}=\mathrm{PG}$ across LVOT after TMVI - PG across LVOT before TMVI, and $\Delta \mathrm{PG}[?] 10 \mathrm{~mm} \mathrm{Hg}$ was defined as significant increase in LVOT gradient according to the Mitral Valve Academic Research Consortium definition. ${ }^{14}$

\subsection{Three-Dimensional transesophageal echocardiography}

Real-time 3D TEE was performed for all patients during TMVI, using an iE33 ultrasound system equipped with a fully sampled matrix-array TEE transducer (X7-2t). 3D images of the MV were acquired with live 3D zoom mode or one beat full volume mode. All images were digitally stored for offline analysis.

Using commercially available software (GI3DQ, QLAB, Philips), the 3D relationship between aortic valve and MV was assessed. First, the end-systolic frame was defined as the last frame during the aortic valve opening. Then, using the 2 orthogonal MV long-axis planes, the MV short-axis cut plane was determined to include the planes of the prosthesis sewing ring, the annuloplasty ring, or the projected native mitral annulus including the posterior saddle-shaped top and the lateral and medial commissures (Figure 1, A-C). The aortic valve long-axis cut plane (Figure 1D, green line; and Figure 1E) was determined to cross both the 
bottom of the right-coronary cusp and the aortic valve commissure between left- and non-coronary cusps. Aortic annular plane was then obtained as the line that connected mitral anterior leaflet insertion point with the bottom of the right-coronary cusp. In patients with aortic valve replacement, the aortic annular plane was carefully determined as the plane perpendicular to the long-axis of the prosthetic valve. 3D derived AM angle was measured as the obtuse angle between the aortic annulus and the MV short-axis (Figure 1F). Post-procedural AM angle, defined as the obtuse angle between the aortic annulus and the transcatheter heart valve short-axis, was also measured following the same steps as above, in which the short-axis cut plane of the transcatheter heart valve was determined to include the plane of the stent valve edge in LA. Notably, the larger the AM angle, the more parallel the LVOT and MV inflow will be and the smaller the angle, the more likely that the MV inflow is directed toward the LVOT and upper septum. We also collected the data of the AM angle measured by MDCT in 26 patients from the medical record, with which the accuracy of the AM angle by $3 \mathrm{D}$ TEE was evaluated.

Using commercially available software (3DQ, QLAB, Philips), the LVOT cross sectional area after stent valve implantation was evaluated from one-beat full volume images including MV and basal LV. The LV long-axis plane in late-systole was determined by following the same process as the determination of the AM angle. A short-axis cut plane (red dotted lines; Figure 2A), perpendicular to the LV long-axis plane, was then moved manually up and down with rotation to search for the smallest area at each level: the level of the valve stent distal edge defined as the most distal portion of the mitral valve stent that was closest to the LV apex and the level of the middle portion of the valve stent (Figure 2, B and C). At both levels, LVOT areas were measured as the area surrounded by the contour of the basal LV wall and the tangent line of the valve stent or the mitral anterior leaflet. Further, the aortic annular area was measured at the level of lowest aortic cusp hinge point (Figure 2D). LVOT dimensions were also measured at each level using LV long-axis cut plane (Figure 2A). In a patient with systolic anterior motion of the anterior mitral leaflet (SAM), LVOT area and dimension at the valve stent distal edge were altered to those at the level of MV leaflet tip (Figure $2, \mathrm{E}-\mathrm{H})$.

\subsection{Observer variability}

To evaluate reliability of measurements, we randomly selected 6 patients. Two independent observers repeated the measurements of the 3D derived pre- and post-procedure AM angle (total 12 variables) and post-procedure LVOT area at valve stent distal edge, middle portion, and aortic annulus (total 18 variables). One observer repeated the measurements at least 1 month later. The intraobserver and interobserver variability was calculated as the absolute differences between the corresponding 2 measurements in the percentage of their mean ( \pm standard deviation) and interclass correlation.

\subsection{Statistical analysis}

Continuous variables were expressed as median (interquartile range), and categorical variables were presented as numbers and percentages. Student paired $t$ test or Wilcoxon signed rank test were used to compare preand post- procedure variables within patients, as appropriate. Comparisons between patients with or without significant increase in LVOT gradient were performed using the student's $t$ test, Wilcoxon rank sum test, or chi-square test, as appropriate. LVOT areas and dimensions among 3 levels: valve stent distal edge, middle portion, and aortic annulus, were compared using one-way analysis of variance with post hocTukey-Kramer testing. The linear regression between continuous variables was made using Pearson's correlation coefficient. For linear correlation, $\triangle \mathrm{PG}$ was log-transformed after adding a constant number (2.0) to the variables to make them positive and non-zero. To identify the independent association with LVOT area at the valve stent distal edge, multivariate linear regression was used among variables including those LV end-systolic volume (LVESV), LV ejection fraction (LVEF), and AM angle which were significantly associated with LVOT area at the valve stent distal edge in univariate analysis. A $\mathrm{P}$ value of $<0.05$ was considered significant. All statistical analyses were performed with JMP 10.0 (SAS Institute Inc., Cary, North Carolina).

\section{RESULTS}

All 43 patients underwent successful TMVI procedure without significant residual regurgitation and stenosis. 
However, one patient with transcatheter mitral ViR implantation developed SAM and severe LVOT obstruction leading to hemodynamic instability. As shown in Table 1, 24 patients (56\%) had TMVI for degenerated bioprosthetic valves, 7 patients $(16 \%)$ had TMVI for failed repair with annuloplasty rings, and 12 patients (28\%) had TMVI for native MV diseases; 8 had severe functional mitral regurgitation, 3 had severe mitral stenosis or regurgitation due to mitral annular calcification, and 1 had rheumatic heart valve. Two patients were implanted balloon-expandable transcatheter stent valves size $23 \mathrm{~mm}, 7$ were implanted stent valves size $26 \mathrm{~mm}$, and the remaining patients (79\%) were implanted stent valves size $29 \mathrm{~mm}$ (Table 2).

\subsection{Echocardiographic measurements before and after TMVI (Table 3)}

At baseline, LV dimensions and basal septum wall thickness were in normal range. However, on average LV volumes were larger and ejection fraction was lower compared to those in normal range. There were 19 patients (44\%) with LV dysfunction (LVEF <50\%), while 6 patients $(14 \%)$ had hyperdynamic LV motion $($ LVEF $>70 \%)$. There was no patient with LVOT obstruction, but there were 2 patients with post aortic valve replacement and elevated peak velocity; one had peak PG of $55 \mathrm{~mm} \mathrm{Hg}$ due to moderate to severe prosthetic valve stenosis, and the other had peak PG of $46 \mathrm{~mm} \mathrm{Hg}$ due to aortic valve prosthesis-patient mismatch.

After the procedure, LV end-diastolic volume and LVEF decreased, while LVESV was not changed. LVOT gradient was significantly increased after TMVI (Figure 3) despite a significant increase in AM angle. One patient with SAM and severe LVOT obstruction had the smallest LVOT area of $0.3 \mathrm{~cm}^{2}$ and increased LVOT gradient of $88 \mathrm{~mm} \mathrm{Hg}$, while pre-procedure LVOT gradient was only $8 \mathrm{~mm} \mathrm{Hg}$ without any evidence of SAM.

\subsection{Change in PG across LVOT after TMVI (Table 4)}

According to the change in LVOT gradient, study patients were classified into 2 groups; patients with significant increase in LVOT gradient defined as $\triangle \mathrm{PG}$ [?]10 mm Hg [n = 10, $\Delta \mathrm{PG}: 22.2$ (13.3-27.1) mm Hg] and those without increase in LVOT gradient defined as $\Delta \mathrm{PG}<10 \mathrm{~mm} \mathrm{Hg}[\mathrm{n}=33, \Delta \mathrm{PG}: 1.7(0-4.4) \mathrm{mm}$ $\mathrm{Hg}$. At baseline, the two groups were similar in age, sex, heart rate, and systolic blood pressure. Device size were not associated with increase in LVOT gradient. Compared to patients without increase in LVOT gradient, patients with increase in LVOT gradient had smaller LV size and AM angle and greater LVEF at pre-procedure, and these differences were also observed at post-procedure.

\subsection{Impact of TMVI on LVOT}

3D derived LVOT area and dimension were evaluated in 29 patients who underwent TMVI. The LVOT area at the level of the valve stent distal edge was newly created after TMVI. As shown in Figure 4, LVOT dimension from LV long-axis cut plane was the minimum at the valve stent distal edge, became larger at the middle portion, and was the largest at the aortic annulus [valve stent distal edge: 9.2 (5.1-15.3), middle portion: 14.0 (11.9-17.8), aortic annulus: $19.2(17.6-20.2) \mathrm{mm} ; \mathrm{P}<0.001]$, while 3D LVOT area tended to large at the valve stent distal edge, gradually became smaller, and was the minimum at the aortic annulus [valve stent distal edge: 7.0 (4.0-11.6), middle portion: 6.0 (4.2-8.1), aortic annulus: $3.9(3.3-4.5) \mathrm{cm}^{2} ; \mathrm{P}<$ 0.001]. Of note, there were 8 patients in whom LVOT area at the level of the aortic annulus was not the minimum; 5 had the minimum area at the valve stent distal edge and the others at the middle portion of the LVOT.

As shown in Figure 5, pre-procedural LVESV, LVEF, and 3D derived AM angle were significantly associated with LVOT area at the valve stent distal edge (LVESV: $\mathrm{r}=0.56, \mathrm{P}=0.001$; LVEF: $\mathrm{r}=-0.57, \mathrm{P}<0.001$; AM angle: $\mathrm{r}=0.68, \mathrm{P}<0.001)$. In addition, post-procedural LVESV, LVEF, and 3D derived AM angle were also associated with that area (post LVESV: $\mathrm{r}=0.49, \mathrm{P}=0.007$; post LVEF: $\mathrm{r}=-0.55, \mathrm{P}=0.002$ : post AM angle: $\mathrm{r}=0.55, \mathrm{P}=0.003)$. Importantly, LVOT area at the valve stent distal edge was significantly smaller in patients with increase in LVOT gradient compared to those without increase in LVOT gradient $(\mathrm{P}<0.001)$ (Table 4), and the correlation between the LVOT area and the [?]PG was strong (Figure 6). Multivariable regression analysis identified that $3 \mathrm{D}$ derived $\mathrm{AM}$ angle was independently associated with LVOT area at the valve stent distal edge (standardized regression coefficient of $0.40, \mathrm{P}=0.017$ ) while LVESV and LVEF 
were not.

\subsection{Observer variability and comparison between 3D TEE and cardiac CT}

The intraobserver variability and intraclass correlation for the measurements of 3D AM angle and LVOT area were $1.5 \pm 1.6 \%$ and $8.9 \pm 7.6 \%$ and 0.96 and 0.98 , respectively. The corresponding interobserver variability values were $1.9 \pm 1.8 \%$ and $9.5 \pm 6.9 \%$ and 0.94 and 0.96 , respectively.

The comparison between 3D TEE and MDCT for the measurement of pre-procedure AM angle was performed. There was a significant although modest correlation between the 2 measurements $(\mathrm{r}=0.62, \mathrm{P}<$ 0.001 ), and Bland-Altman showed the systematic difference was $2.4^{\circ}$ with limits of agreement of $\pm 11.7^{\circ}$.

\section{DISCUSSION}

This is the first study to assess the newly created LVOT cross sectional area after TMVI using 3D TEE. 3D assessment of cross-sectional image directly shows the anatomical relationship among the stent valve, mitral leaflet, and basal LV wall after TMVI. Moreover, the current study shows that narrowed LVOT area at the valve stent distal (ventricular side) edge depends on small LV size, preserved LVEF, and small 3D derived aorto-mitral angle. Only real-time 3D TEE can identify the relationship between the transcatheter heart valve and basal LV with precise measurements of LVOT area at any level during the procedure.

\subsection{Cause of LVOT narrowing in patients undergoing transcatheter ViV implantation}

In patients with degenerated bioprosthetic valves, who do not have their native mitral valve, the stent valve itself encroaches into LVOT space and alters the LVOT geometry and flow. The causes responsible for LVOT obstruction appear to be multifactorial. Previously, LVOT obstruction after surgical mitral valve replacement has been identified when the valve was implanted in a small ventricular cavity or hypertrophic left ventricle. ${ }^{15}$ The results of the current study also showed that small LV size with preserved LV contraction and small AM angle before procedure might decrease LVOT area at the valve stent distal edge and increase LVOT gradient after transcatheter mitral ViV implantation, because the stent valve implanted in the mitral position with small AM angle could protrude into the outflow tract near the basal septum especially in patients with small LV cavity.

\subsection{Cause of LVOT narrowing in patients undergoing transcatheter ViR or ViN implantation}

LVOT obstruction may more commonly occur in ViR or ViN than in ViV implantation because, in addition to the valve encroachment, the native redundant mitral leaflet can cause SAM. Previously, LVOT obstruction with SAM has been described in patients undergoing MV repair for myxomatous MV prolapse. ${ }^{16}$ The large posterior leaflet and the reduction of the anteroposterior diameter using an annuloplasty ring cause the leaflets to coapt anteriorly, increasing the likelihood of SAM and LVOT obstruction. ${ }^{17}$ Ro et al. reported that the angle of systolic flow directed onto the posterior surface of the mitral leaflet was important to produce the pushing force (drag force), resulting initially in SAM and later the Venturi effect. ${ }^{18}$ Similarly, in patients undergoing ViV or ViN procedure, valve implantation in the mitral position with small AM angle can cause displacement of the mitral anterior leaflet anteriorly toward LVOT, making the leaflet more susceptible to the effect of systolic outflow and receiving the drag force and Venturi effect.

\subsection{The role of 3D TEE compared to MDCT}

Recent valvular heart disease guidelines and literature have recognized the importance of advanced cardiovascular imaging, especially 3D TEE and cardiac CT. ${ }^{7,8,19}$ MDCT provides a 3D dataset with good to excellent visualization of anatomic details of the MV and LVOT, enabling us to analyze the AM angle and predicted neo-LVOT area, which seems to contribute to the assessment of LVOT obstruction risk as well as patient selection, planning, and valve sizing for TMVI procedure. ${ }^{9,20}$ On the other hand, echocardiography including 3D TEE could be an alternative to MDCT in such patients as those with unstable clinical status, known allergy to iodinated contrast, kidney disease, tachycardia, and unable to hold their breath. Our results contribute to the risk stratification of the increase in LVOT gradient after procedure. More importantly, TMVI cannot be performed without real-time 2D and 3D TEE as it provides guidance for transseptal puncture and 
crossing, alignment of the delivery system using either a 3D en facesurgical view or a simultaneous multiplane (orthogonal) 2D view, and assessment for perivalvular leakage and LVOT obstruction after TMVI. ${ }^{7,8,21}$ Furthermore, analysis of newly created LVOT area by real-time 3D TEE just after deployment of the stent valve in the catheterization-lab not only confirms the success of TMVI but also may predict future LVOT obstruction.

\subsection{The role of $3 \mathrm{D}$ TEE compared to $2 \mathrm{D}$ TEE}

In a clinical setting, we used to suspect LVOT obstruction when we saw the encroachment of the stent valve toward LVOT space by 2D LV long-axis views. Also, we've frequently experienced that a stent valve may severely encroach into the LVOT without significant subsequent elevation of LVOT velocity. This apparent discrepancy may be explained by this study; the dimension between the stent struts and ventricular septum from 2D LV long-axis view was the minimum at the valve stent distal edge, while 3D derived LVOT cross sectional area was typically the maximum at the valve stent distal edge (Figure 4). Our study suggests the need of the 3D TEE if LVOT obstruction is suspected during or after the procedure by 2D LV long-axis view.

\subsection{Assessment of narrowed LVOT using Doppler echocardiography}

The assessment of LVOT narrowing using continuous wave Doppler is problematic because the measurement of the peak velocity or PG often has technical difficulties, including Doppler angulation and inaccuracy of the location of the smallest area. ${ }^{22}$ To mitigate these problems, instead of peak PG, we analyzed the change in PG from before to after procedure. The reason why LVOT gradient was increased even though the smallest area was not dramatically changed after TMVI may be due to the following: the valve implantation newly creates a narrowed LVOT surrounded by the valve and left ventricular septum, which potentially causes a tunnel effect increasing the blood flow velocity compared to the pre-procedural condition. ${ }^{23}$ Thus, the change or increase of peak velocity and PG ([?]PG) reflects the newly created LVOT geometry after TMVI, and, for that reason, an increase in LVOT gradient by more than $10 \mathrm{~mm} \mathrm{Hg}$ was used to define LVOT obstruction. ${ }^{14}$ However, further study will be required to show whether this cut-off value is reasonable and clinically meaningful as definition.

\subsection{Study limitations}

Data for this study were collected retrospectively from single center database. Accordingly, our study included some limitations. First, there were the numerous factors influencing our results such as the sample size as well as the risk for selection bias and the observer bias. Our study sample size was small, especially patients who had significant increase in LVOT gradient after the TMVI, leading to the statistical limitation to examine the impact of variables on LVOT narrowing. In addition, the population was very heterogeneous in terms of TMVI settings (ViV, ViR, and ViN). Further, the measurer could not measure all the parameters blindly because the measurer knew that a prosthetic valve was placed. Second, the current study evaluated the LVOT narrowing after the balloon-expandable transcatheter aortic valves implantation in the mitral position. The data would differ if a different transcatheter mitral valve was used.

\section{CONCLUSION}

Left ventricular size, systolic function, and 3D derived aorto-mitral angle were associated with LVOT narrowing in patients undergoing transcatheter mitral valve implantation. 3D TEE can directly assess newly created LVOT with its unique morphological shape, which may help predict the risk of an increased LVOT gradient after TMVI.

\section{Acknowledgements}

We thank Shusuke Kijima and Misa Morita, from Philips Electronics Japan, for their technical assistance.

\section{Funding}


This research did not receive any specific grant from funding agencies in the public, commercial, or non-forprofit sectors.

\section{Author contributions}

Concept/design, Data analysis/interpretation, Drafting article, Critical revision of article, Approval of article, Statistics, Funding secured by, Data collection, Other.

\section{References}

1. Seiffert M, Conradi L, Baldus S, et al. Transcatheter mitral valve-in-valve implantation in patients with degenerated bioprostheses. JACC Cardiovasc Interv. 2012;5:341-349.

2. Petronio A, Giannini C, De Carlo M, et al. Antegrade percutaneous valve implantation for mitral ring dysfunction, a challenging case. Catheter Cardiovasc Interv. 2012;80:700-703.

3. Eleid MF, Cabalka AK, Williams MR, et al. Percutaneous transvenous transseptal transcatheter valve implantation in failed bioprosthetic mitral valves, ring annuloplasty, and severe mitral annular calcification. JACC Cardiovasc Interv. 2016;9:1161-1174.

4. Yoon SH, Whisenant BK, Bleiziffer S, et al. Transcatheter mitral valve replacement for degenerated bioprosthetic valves and failed annuloplasty rings. J Am Coll Cardiol. 2017;70:1121-1131.

5. Guerrero M, Urena M, Himbert D, et al. 1-Year Outcomes of Transcatheter Mitral Valve Replacement in Patients With Severe Mitral Annular Calcification. J Am Coll Cardiol. 2018;71:1841-1853.

6. Bouleti C, Fassa AA, Himbert D, et al. Transfemoral implantation of transcatheter heart valves after deterioration of mitral bioprosthesis or previous ring annuloplasty. JACC Cardiovasc Interv.2015;8:83-91.

7. Mackensen GB, Lee JC, Wang DD, et al. Role of Echocardiography in Transcatheter Mitral Valve Replacement in Native Mitral Valves and Mitral Rings. J Am Soc Echocardiogr. 2018;31:475-490.

8. Mankad SV, Aldea GS, Ho NM, et al. Transcatheter Mitral Valve Implantation in Degenerated Bioprosthetic Valves. J Am Soc Echocardiogr. 2018;31:845-859.

9. Blanke P, Naoum C, Dvir D, et al. Predicting LVOT obstruction in transcatheter mitral valve implantation: concept of the neo-LVOT. JACC Cardiovasc Imaging. 2017;10:482-485.

10. Yoon SH, Bleiziffer S, Latib A, et al. Predictors of Left Ventricular Outflow Tract Obstruction After Transcatheter Mitral Valve Replacement. JACC Cardiovasc Interv. 2019;12:182-193.

11. Nampiaparampil RG, Swistel DG, Schlame M, et al. Intraoperative Two- and Three-Dimensional Transesophageal Echocardiography in Combined Myectomy-Mitral Operations for Hypertrophic Cardiomyopathy. J Am Soc Echocardiogr. 2018;31:275-288.

12. Mihara H, Shibayama K, Harada K, et al. LV outflow tract area in discrete subaortic stenosis and hypertrophic obstructive cardiomyopathy: a real-time 3-dimensional transesophageal echocardiography study. JACC Cardiovasc Imaging. 2014;7:425-428.

13. Mitchell C, Rahko PS, Blauwet LA, et al. Guidelines for Performing a Comprehensive Transthoracic Echocardiographic Examination in Adults: Recommendations from the American Society of Echocardiography. J Am Soc Echocardiogr. 2019;32:1-64.

14. Stone GW, Adams DH, Abraham WT, et al. Clinical Trial Design Principles and Endpoint Definitions for Transcatheter Mitral Valve Repair and Replacement: Part 2: Endpoint Definitions: A Consensus Document From the Mitral Valve Academic Research Consortium. J Am Coll Cardiol. 2015;66:308-321.

15. Jett GK, Jett MD, Barnhart GR, et al. Left ventricular outflow tract obstruction with mitral valve replacement in small ventricular cavities. Ann Thorac Surg. 1986;41:70-74. 
16. Galler M, Kronzon I, Slater J, et al. Long-term follow-up after mitral valve reconstruction: incidence of postoperative left ventricular outflow obstruction. Circulation. 1986;74:I99-103.

17. Maslow AD, Regan MM, Haering JM, et al. Echocardiographic predictors of left ventricular outflow tract obstruction and systolic anterior motion of the mitral valve after mitral valve reconstruction for myxomatous valve disease. J Am Coll Cardiol. 1999;34:2096-2104.

18. Ro R, Halpern D, Sahn DJ, et al. Vector flow mapping in obstructive hypertrophic cardiomyopathy to assess the relationship of early systolic left ventricular flow and the mitral valve. J Am Coll Cardiol.2014;64:1984-1995.

19. Blanke P, Naoum C, Webb J, et al. Multimodality imaging in the context of transcatheter mitral valve replacement: establishing consensus among modalities and disciplines. JACC Cardiovasc Imaging. 2015;8:1191-1208.

20. Naoum C, Blanke P, Cavalcante JL, et al. Cardiac computed tomography and magnetic resonance imaging in the evaluation of mitral and tricuspid valve disease: implications for transcatheter interventions. Circ Cardiovasc Imaging. 2017;10:1-17.

21. Nyman CB, Mackensen GB, Jelacic S, et al. Transcatheter mitral valve repair using the edge-to-edge clip. J Am Soc Echocardiogr.2018;31:434-453.

22. Baumgartner H, Hung J, Bermejo J, et al. Recommendations on the echocardiographic assessment of aortic valve stenosis: a focused update from the european association of cardiovascular imaging and the american society of echocardiography. J Am Soc Echocardiogr.2017;30:372-392.

23. Maron BJ, Redwood DR, Roberts WC, et al. Tunnel subaortic stenosis: left ventricular outflow tract obstruction produced by fibromuscular tubular narrowing. Circulation. 1976;54:404-416.

\section{Figure legends}

Figure 1. Measurement of aorto-mitral (AM) angle in patient with native MV disease. Using the 2 orthogonal MV long-axis planes, the MV short-axis cut plane was determined to include the posterior saddle-shaped top and the lateral and medial commissures (Panel A-C). The AV long-axis cut plane was then obtained to include the aortic valve commissure between left- and non-coronary cusps (Panel D, green line) and the center of the right-coronary cusp. In this process, the MV short-axis cut plane was moved to obtain the aortic valve commissure and rotated to search for the bottom of the right-coronary cusp (= the center of the right-coronary cusp) in the AV long-axis cut plane (Panel E). The MV anterior leaflet insertion point on the AV long-axis cut plane was determined by visual identification using the cine loop. Finally, aortic annulus was obtained as the line that connected the mitral anterior leaflet insertion point with the bottom of the right-coronary cusp. 3D derived AM angle was measured as the obtuse angle between the aortic annulus and the MV short-axis (Panel F).

AV indicates aortic valve; LA, left atrium; LV, left ventricle; and MV, mitral valve.

Figure 2. Planimetry of the LVOT cross sectional area. Panel A shows the left ventricular long-axis plane. Transcatheter heart valve (green arrows) encroaches into LVOT. Panel B and C are the cross sectional shortaxis planes with orientation indicated by red dotted lines [Panel A, (B) and (C)] at the valve stent distal edge and middle portion of the valve stent. The short-axis cut plane is moved manually up and down with rotation to search for the smallest area at each level. Importantly, care was taken that this area should not be moved from the plane perpendicular to the LV outflow long-axis. Panel D shows the short-axis image of the aortic annulus identified at the level of lowest aortic cusp hinge point. LVOT dimension and cross sectional area are measured at 3 levels (d1-d3 and a1-a3). In a patient with systolic anterior motion of mitral leaflet, who has the narrowest LVOT area at the level of leaflet tip, LVOT dimension and area at the valve stent distal edge are altered to these at the leaflet tip (Panel E-H). The LVOT area at the level of the leaflet tip is $0.3 \mathrm{~cm}^{2}$, surrounded by the anterior mitral leaflet (white arrowheads) and ventricular septum (yellow arrowheads). 
Abbreviations as in Figure 1.

Calibration makers were shown on Figure A (for Figures A through D) and E (for Figures E through H).

Figure 3. Change in pressure gradient across LVOT in study patients.

LVOT indicates left ventricular outflow tract; and PG, pressure gradient.

Figure 4. The LVOT dimensions and cross sectional areas at 3 levels including the valve stent distal edge, middle portion of the valve stent, and aortic annulus. The LVOT dimension gradually became larger from the level at the valve stent distal edge to the aortic annulus, while the LVOT cross sectional area gradually became smaller from the level at the valve stent distal edge to the aortic annulus. Of note, there were 9 patients in whom LVOT area at the aortic annulus was not the minimum; 5 have the minimum area at the valve stent distal edge (red lines) and the others at the middle portion (green lines).

LVOT indicates left ventricular outflow tract.

*the most distal portion of the mitral valve stent that was closest to the LV apex

Figure 5. Relationships between LVOT cross sectional area at the valve stent distal edge versus LVESV, LVEF, and aorto-mitral angle. The correlations between the area and LVESV, LVEF, and aorto-mitral angle were moderate.

LVEF indicates left ventricular ejection fraction; LVESV, and left ventricular end-systolic volume.

Figure 6. Correlation between LVOT cross sectional area at the valve stent distal edge and the change in LVOT gradient.

[?]PG indicates change in pressure gradient across LVOT.

\section{Tables}

Table 1 Baseline characteristics $(n=43)$

\begin{tabular}{ll}
\hline Age, years & $73(68-81)$ \\
\hline Male & $16(36)$ \\
BSA, m ${ }^{2}$ & $1.83(1.67-1.99)$ \\
Heart rate, bpm & $72(59-83)$ \\
Systolic blood pressure, mmHg & $122(111-138)$ \\
Diastolic blood pressure, mmHg & $64(59-74)$ \\
Atrial fibrillation & $21(50)$ \\
Ischemic heart disease & $24(56)$ \\
TMVI setting & $24(56)$ \\
Degenerated bioprosthetic valve & $7(16)$ \\
Failed repair with annuloplasty ring & $12(28)$ \\
Native mitral valve disease &
\end{tabular}

Values are median (interquartile range) or $\mathrm{n}(\%)$.

BSA indicates body surface area; and TMVI, transcatheter mitral valve implantation.

Table 2 Device type and size

\begin{tabular}{llllll}
\hline & Device size & Device size & Device size & Device size & Device size \\
\hline Device type & $23 \mathrm{~mm}$ & $26 \mathrm{~mm}$ & $29 \mathrm{~mm}$ & total \\
& Sapien XT & 0 & 2 & 3 & $5(12)$ \\
& Sapien 3 & 2 & 5 & 31 & $38(88)$
\end{tabular}




\begin{tabular}{lllll}
\hline Device size & Device size & Device size & Device size & Device size \\
\hline total & $2(5)$ & $7(16)$ & $34(79)$ & $43(100)$ \\
\hline
\end{tabular}

Values are $\mathrm{n}(\%)$.

Table 3 Echocardiographic measurements of study patients $(n=43)$

\begin{tabular}{lll}
\hline & Pre-procedure & Post-procedure \\
\hline LVDd, mm & $52(44-60)$ & $51(42-57)$ \\
LVDs, mm & $34(27-50)$ & $35(28-49)$ \\
IVS thickness, mm & $10(8-12)$ & $11(9-12)$ \\
IVS wall motion & & \\
Normal or hyperkinesis & $23(53)$ & $23(53)$ \\
Hypokinesis or akinesis & $20(47)$ & $20(47)$ \\
LVEDV, ml & $156(106-185)$ & $146(117-180)^{*}$ \\
LVESV, ml & $74(37-102)$ & $80(37-115)$ \\
LVEF, \% $_{\text {Aorto-mitral angle, }}{ }^{\circ}$ & $52(39-65)$ & $48(34-68)^{*}$ \\
Pressure gradient across LVOT, mmHg & $126(118-131)$ & $128(122-143)^{*}$ \\
\hline
\end{tabular}

Values are median (interquartile range) or $\mathrm{n}(\%) .{ }^{*} \mathrm{p}<0.05$, vs Pre-procedure.

IVS indicates inter ventricular septum; LVDd, left ventricular end-diastolic dimension; LVDs, left ventricular end-systolic dimension; LVEDV, left ventricular end-diastolic volume; LVEF, left ventricular ejection fraction; LVESV, left ventricular end-systolic volume; and LVOT, left ventricular outflow tract.

Table 4 Comparison between patients with and without increase of PG

\begin{tabular}{|c|c|c|c|}
\hline & $\Delta \mathrm{PG}<10(\mathrm{n}=33)$ & $\Delta \mathrm{PG}[?] 10(\mathrm{n}=10)$ & $\mathrm{p}$ Value \\
\hline Age, years & $72(67-80)$ & $76(65-83)$ & 0.47 \\
\hline Male & $12(36)$ & $4(40)$ & 0.83 \\
\hline Heart rate, bpm & $72(63-84)$ & $68(55-81)$ & 0.37 \\
\hline $\begin{array}{l}\text { Systolic blood pressure, } \\
\mathrm{mmHg}\end{array}$ & $123(112-138)$ & $115(104-128)$ & 0.20 \\
\hline $\begin{array}{l}\text { Diastolic blood } \\
\text { pressure, } \mathrm{mmHg}\end{array}$ & $65(60-78)$ & $55(44-63)$ & 0.003 \\
\hline TMVI setting & & & 0.76 \\
\hline $\begin{array}{l}\text { Degenerated } \\
\text { bioprosthetic valve }\end{array}$ & $18(55)$ & $6(60)$ & \\
\hline $\begin{array}{l}\text { Failed repair with } \\
\text { annuloplasty ring or } \\
\text { native mitral valve } \\
\text { disease }\end{array}$ & $15(45)$ & $4(40)$ & \\
\hline Device size & & & 0.32 \\
\hline $23 \mathrm{~mm}$ & $2(6)$ & $0(0)$ & \\
\hline $26 \mathrm{~mm}$ & $4(12)$ & $3(30)$ & \\
\hline $\begin{array}{l}29 \mathrm{~mm} \\
\text { Echocardiography } \\
\text { measurements }\end{array}$ & $27(82)$ & $7(70)$ & \\
\hline
\end{tabular}




\begin{tabular}{|c|c|c|c|}
\hline & $\Delta \mathrm{PG}<10(\mathrm{n}=33)$ & $\Delta \mathrm{PG}[?] 10(\mathrm{n}=10)$ & $\mathrm{p}$ Value \\
\hline \multicolumn{4}{|l|}{ Pre-procedure } \\
\hline LVEDV, ml & $163(120-196)$ & $105(87-171)$ & 0.01 \\
\hline LVESV, ml & $87(54-117)$ & $37(20-67)$ & 0.001 \\
\hline LVEF, \% & $44(36-58)$ & $66(58-78)$ & $<0.001$ \\
\hline Aorto-mitral angle, ${ }^{\circ}$ & $126(121-134)$ & $116(111-120)$ & 0.001 \\
\hline $\begin{array}{l}\text { Pressure gradient } \\
\text { across LVOT, } \mathrm{mmHg}\end{array}$ & $7.8(4.2-16.0)$ & $13.0(10.4-26.0)$ & 0.06 \\
\hline \multicolumn{4}{|l|}{ Post-procedure } \\
\hline LVEDV, ml & $160(124-181)$ & $116(82-148)$ & 0.01 \\
\hline LVESV, ml & $95(50-122)$ & $35(22-70)$ & 0.004 \\
\hline LVEF, \% & $42(32-58)$ & $69(52-72)$ & 0.001 \\
\hline Aorto-mitral angle, ${ }^{\circ}$ & $131(126-144)$ & $118(113-125)$ & $<0.001$ \\
\hline $\begin{array}{l}\text { Pressure gradient } \\
\text { across LVOT, mmHg }\end{array}$ & $10.2(6.8-19.4)$ & $36.0(26.5-59.6)$ & $<0.001$ \\
\hline LVOT area*, $\mathrm{cm}^{2}$ & $9.6(6.0-14.1)$ & $3.4(1.6-5.3)$ & $<0.001$ \\
\hline
\end{tabular}

Values are median (interquartile range) or $\mathrm{n}(\%)$.

LVEDV indicates left ventricular end-diastolic volume; LVEF, left ventricular ejection fraction; LVESV, left ventricular end-systolic volume; LVOT, left ventricular outflow tract; TMVI, transcatheter mitral valve implantation; and [?]PG, change in pressure gradient across LVOT.

*LVOT area measured at the valve stent ventricular edge.

\section{Figure 1.}

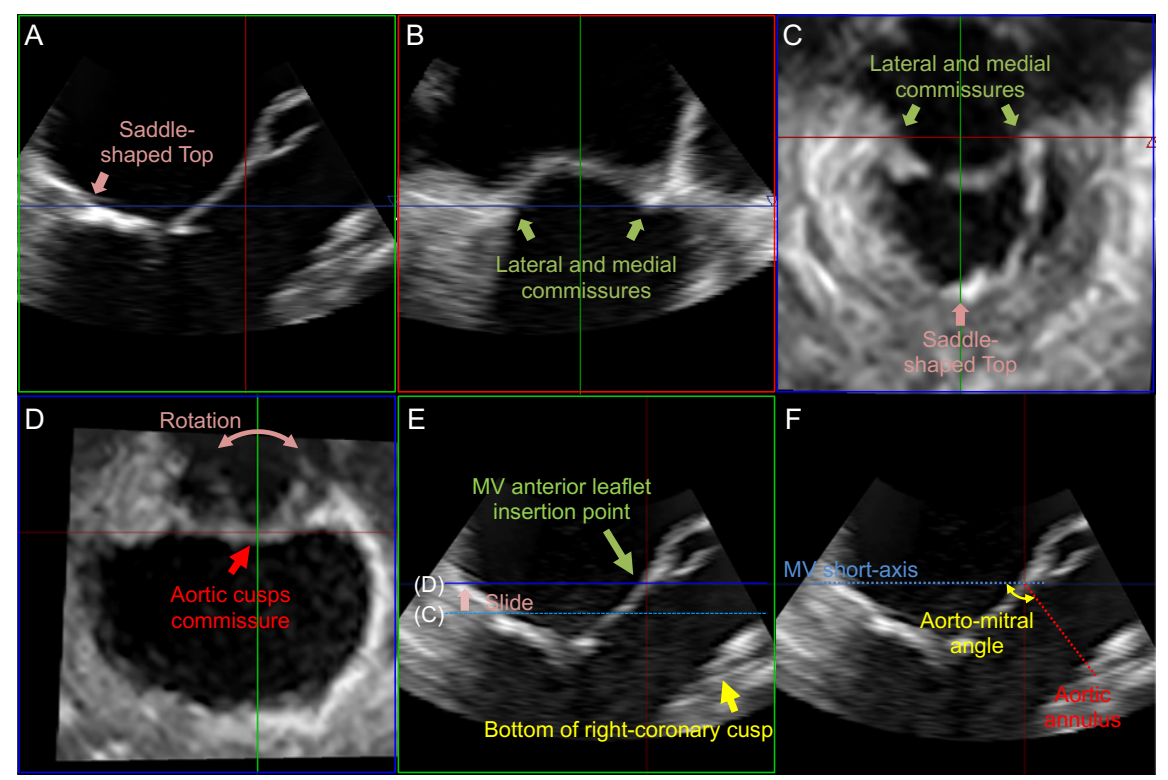


Figure 2.

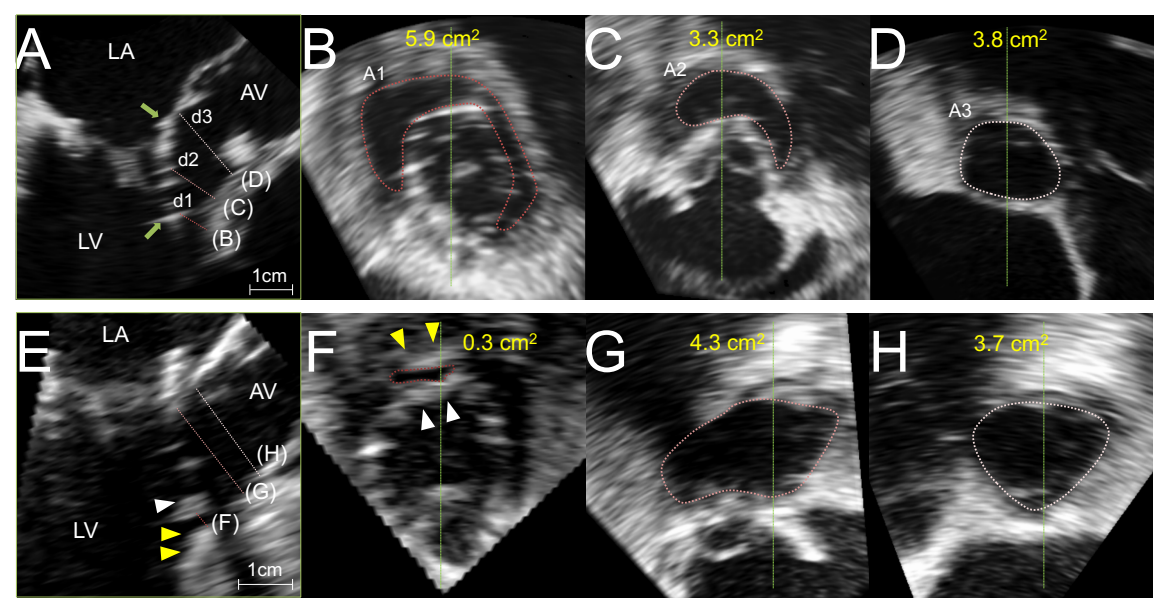

Figure 3.

PG across LVOT

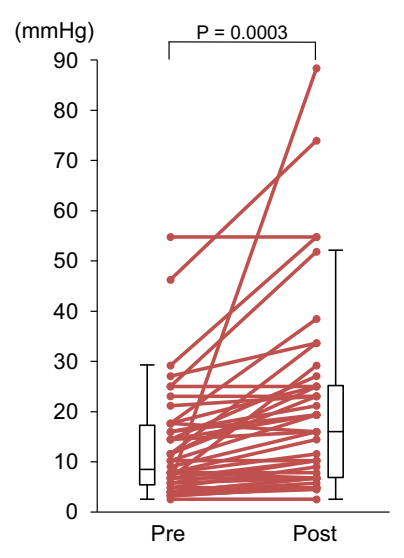




\section{Figure 4.}
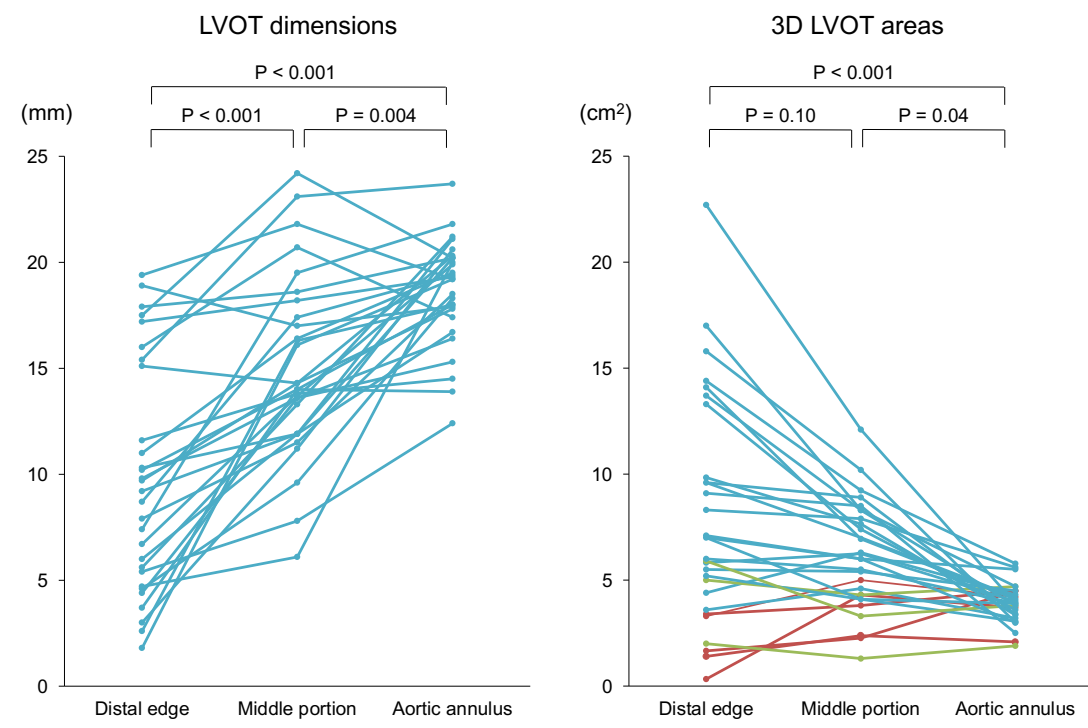

Figure 5.
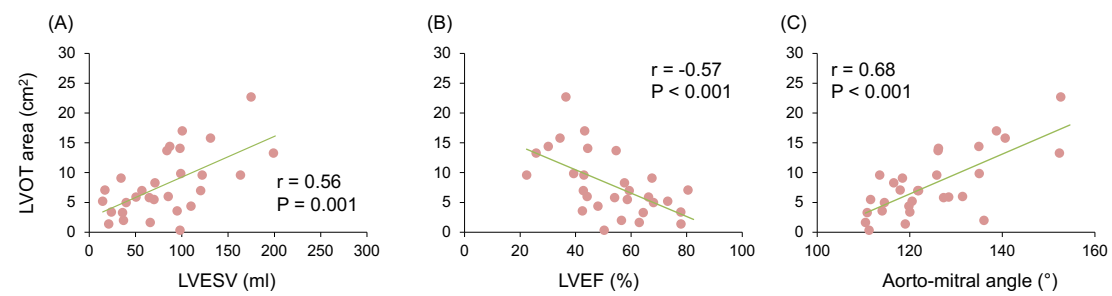
Figure 6.

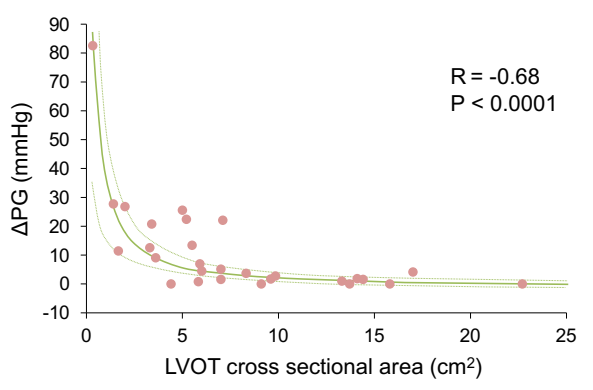

